BNL-114560-2017-JA

\title{
Finding a Needle in the Haystack: Identification of Functionally Important Minority Phases in Operating Battery
}

\author{
K. Zhang, X. Wang
}

Submitted to Nano Letters

November 2017

Chemistry Department

Brookhaven National Laboratory

\section{U.S. Department of Energy \\ USDOE Office of Energy Efficiency and Renewable Energy (EERE)}




\section{DISCLAIMER}

This report was prepared as an account of work sponsored by an agency of the United States Government. Neither the United States Government nor any agency thereof, nor any of their employees, nor any of their contractors, subcontractors, or their employees, makes any warranty, express or implied, or assumes any legal liability or responsibility for the accuracy, completeness, or any third party's use or the results of such use of any information, apparatus, product, or process disclosed, or represents that its use would not infringe privately owned rights. Reference herein to any specific commercial product, process, or service by trade name, trademark, manufacturer, or otherwise, does not necessarily constitute or imply its endorsement, recommendation, or favoring by the United States Government or any agency thereof or its contractors or subcontractors. The views and opinions of authors expressed herein do not necessarily state or reflect those of the United States Government or any agency thereof. 


\section{Finding a Needle in the Haystack: Identification of Functionally Important Minority Phases in Operating Battery}

Kai Zhang ${ }^{\dagger, \star,}$, Fang Ren ${ }^{\ddagger}$, , Xuelong Wang ${ }^{\S, /,}$, , Enyuan $\mathrm{Hu}^{\S}$, Yahong Xu ${ }^{\ddagger}$, Xiao-Qing Yang ${ }^{\S}$, Hong Li", Liquan Chen", Piero Pianetta ${ }^{\ddagger}$, Apurva Mehta ${ }^{\ddagger}$, , Xiqian Yu",*, Yijin Liu ${ }^{\ddagger}$,

${ }^{\dagger}$ Beijing Synchrotron Radiation Facility, Institute of High Energy Physics, Chinese Academy of

Science, Beijing 100049, China

${ }^{\ddagger}$ Stanford Synchrotron Radiation Lightsource, SLAC National Accelerator Laboratory, Menlo Park, CA 94025, USA

${ }^{\S}$ Chemistry Division, Brookhaven National Laboratory, Upton, NY 11973, USA

"Beijing National Laboratory for Condensed Matter Physics, Institute of Physics, Chinese Academy of Sciences, Beijing 100190, China

${ }^{\perp}$ These authors contributed equally to this work.

*Correspondence and requests for materials should be addressed to A.M. (mehta@slac.stanford.edu),X.Y. (xyu@iphy.ac.cn), Y.L. (liuyijin@slac.stanford.edu)

\section{Abstract}

The in-depth understanding of the minority phases' roles in functional materials, e.g. batteries, is critical for optimizing the system performance and the operational efficiency. Although the visualization of battery electrode under operating conditions has been demonstrated, the development of advanced data-mining approaches is still needed in order to identify minority phases and to understand their functionality. The present study uses nanoscale x-ray spectromicroscopy to study a functional $\mathrm{LiCoO}_{2} / \mathrm{Li}$ battery pouch cell. The data-mining approaches developed herein were used to search through over 10 million X-ray spectra that cover more than 100 active cathode particles. Two particles with unanticipated chemical fingerprints were identified and further analyzed, providing direct evidence and valuable insight into the undesired side reactions involving the cation dissolution and precipitation as well as the local overlithiation caused sub-particle domain deactivation. The data-mining approach described in this work is widely applicable to many other structurally complex and chemically heterogeneous systems, in which the secondary/minority phases could critically affect the overall performance of the system, well beyond battery research.

Keywords: Li-ion battery; Minority phase; X-ray spectro-microscopy; Data mining; Clustering algorithm.

Secondary phases are ubiquitous in functional materials and devices that operate under real world conditions, which provide the reaction driving force that governs the formation and evolution of the secondary phases. The secondary phases are often intermediates phases as the material undergoes transformation; in such cases they are anticipated, but their location/distribution is unknown. Sometimes, however, these secondary phases are unanticipated; they are side-products, unknown intermediates, or phases that exist under conditions not 
anticipated in routine operations. Discovery, identification and mapping the distribution of these secondary phases is therefore frequently at the core of true understanding of how materials and devices work in the real world. The secondary phases, however, are usually small in their total population and are often sparse in their spatial distribution (namely the minority phase for this type). They could even be metastable meaning that some of them only exist in conditions that are away from equilibrium. These characteristics make it very difficult to find and study them in hierarchically complex and chemically heterogeneous systems. Examples of the functionally important minority phases include but are not limited to (1) undesired phase transformation on the surface of battery electrode $\mathrm{LiNi}_{\mathrm{x}} \mathrm{Mn}_{\mathrm{x}} \mathrm{Co}_{1-2 \mathrm{x}} \mathrm{O}_{2}$ (from a layered structure to a rock-salt structure) that affects the ionic conductivity of the particle surface and, thus, redirects the reaction pathways ${ }^{1}$; (2) the formation of localized large chemical gradient in $\mathrm{Li}_{1+\mathrm{x}} \mathrm{Mn}_{2-\mathrm{x}} \mathrm{O}_{4}$ upon electrochemical cycling, which is responsible for mechanical strain that leads to the formation and propagation of cracks in the single crystal particle ${ }^{2}$; (3) accumulation of poisoning metals on the surface of the catalytic particles, which affects their efficiency in petroleum refining through reducing the accessibility of the internal active domains ${ }^{3}$. As shown in these examples, the minority phases determine the overall system performance and functionality by critically influencing the overall behavior of the materials. Some of the secondary phases are actively involved in the reaction bringing in grain boundaries and interfaces that could affect the overall transport property of the material ${ }^{4}$; others may be inactive and even spatially isolated, however, affecting the bulk property through acting as physical barriers.

Locating, identifying and characterizing minority phases is challenging for several reasons. In bulk measurements like x-ray spectroscopy, x-ray diffraction and Raman spectroscopy, the signal from the majority phase dominates and masks the weak signal from the minority phase. The detection of minority phases, when they are metastable or only appear under nonequilibrium operation conditions, requires the measurement to be performed in-situ and the data to be acquired within reasonable time frame, which complicates the experimental design. The sparse spatial distribution of the minority phases usually requires surveying large sample using fine probes that can provide sufficient spatial resolution. Such requirement is already challenging from the experimental point of view, not to mention the difficulties associated with finding and analyzing the scientifically important constituents of the large scale experimental data.

The importance of locating minority phases in obtaining a deeper understanding of complex working materials and devices has driven the rapid development of fast, high-resolution, chemically sensitive probes, especially at large scale experimental facilities such as synchrotron lightsources, capable of capturing material changes under operando conditions. In addition to the examples discussed above, we have seen more intense research activities attempting to capture the signatures of the minority phases. Zhou et al. performed time resolved operando x-ray diffraction (XRD) on an operating battery to investigate the intermediate phase that forms when the $\mathrm{LiNi}_{1 / 3} \mathrm{Mn}_{1 / 3} \mathrm{Co}_{1 / 3} \mathrm{O}_{2}$ electrode was cycled at fast rate ${ }^{5}$. Duan et al. combined nanoscale $\mathrm{x}$-ray spectro-microscopy with an unsupervised data clustering algorithm to reveal the surface corrosion effect of rare-earth permanent magnet ${ }^{6}$. Lim et al. employed soft x-ray STXM 
(scanning transmission $\mathrm{x}$-ray microscopy) technique to image many $\mathrm{LiFePO}_{4}$ particles and revealed the reaction pathways that are strongly dependent on the cycling rate ${ }^{7}$. These studies clearly show the strength of synchrotron based experimental techniques, while highlighting the need for the extraction of scientifically important information through data-mining approaches.

As discussed, in order to take full advantage of the advances in synchrotron based experimental techniques, it is important develop computing methods that can effectively extract scientifically important information from the data. We choose to study the Li ion battery electrode material in this work for demonstration of the application of advanced data attribute extraction methods and clustering methods in synchrotron based nanoscale x-ray spectro-microscopy. Synchrotron based spectro-microscopy has been widely used in the studies of Li ion battery electrode materials ${ }^{7-13}$. In our earlier work ${ }^{14}$, a battery pouch cell setup with an x-ray transparent window was designed and constructed to perform synchrotron based in-situ nanoscale spectro-microscopic study of $\mathrm{LiCoO}_{2}$ cathode particles' behavior over long term cycling. We monitored an active particle over many electro-chemical cycles at different rates ${ }^{14}$. With prior knowledge of the spectroscopic signatures of $\mathrm{LiCoO}_{2}$ at the charged states (at $4.6 \mathrm{~V}$ ) and the discharged stages (at $3 \mathrm{~V}$ ), we were able to visualize the evolution of the sub-particle level state-of-charge heterogeneity, which provided critical insights into the active particle's response to different local electro-chemical environments that are governed by the cycling rate and history.

Although these results very nicely demonstrated the strength of the synchrotron based nanoscale spectro-microscopy technique for battery research ${ }^{15}, 16$, we would point out that there is a limitation in the above described "supervised" approach. In earlier works, there is a heavy reliance on the prior knowledge of a list of chemical components that are anticipated to exist in the sample under investigation. The quantification and interpretation of the spectro-microscopic data are usually carried out by comparing the experimentally measured spectra with that of the anticipated chemical species. Linear combination fitting is widely used in this field to provide an evaluation of the local chemical composition based on the similarity in the spectroscopic fingerprints ${ }^{16}$. This approach is straightforward and very efficient. However, it is limited by anticipated species defined by the prior knowledge and, unavoidably, misses the opportunity to discover unanticipated chemical compounds. We would point out that, when specific attention is paid in the process of the data, it is possible to retrieve some of the missing information ${ }^{17}$. However, more robust and advanced computing methods are clearly needed in order to identify and study unanticipated minority phases that could be functionally important for reasons discussed above.

Herein, we present data-mining aided nanoscale spectro-microscopic study of $\mathrm{LiCoO}_{2}$ electrode in a functional battery cell. After the battery cell has gone through a designed cycling sequence ${ }^{14}$, we use the chemically sensitive x-ray probe to survey a large area over the battery pouch covering more than 100 active particles. We first extracted several data attributes from the spectra resulting in the spatial distributions of these key descriptors of the spectroscopic fingerprints. Data clustering algorithms are then applied to the data attribute maps, which lead to 
the identification of particles with distinct chemical signature. Out of the more than 100 active particles covered in our scan, we identified two of them that show unanticipated reduced Co valence states. The discovered minority phases (these two unexpected particles) are further analyzed and are attributed to different side reactions occurred in the cell during the electrochemical cycling. Our findings provide direct evidence showing that the Co dissolution/precipitation and mechanical failure (due to local over-lithiation herein) are both important degradation mechanisms that are responsible for the capacity fade of $\mathrm{LiCoO}_{2}$ upon electrochemical cycling. Beyond battery research, we anticipate that the data-mining approach presented in this work is applicable to a large number of research fields, in which the secondary/minority phases play important roles in affecting the overall performance of the functionally materials and devices.

As discussed above, often the important phases in functional materials and devices, including the $\mathrm{LiCoO}_{2}$ battery cell, are unanticipated minority phases. These minority phases are often unknown and, thus, cannot be included as prior knowledge to enable the supervised $\operatorname{search}^{16}$. In order to facilitate the study of the unanticipated minority phases formed in the battery cell upon operation, we surveyed the cell (Figure 1.d) using the synchrotron based transmission x-ray microscope (Figure 1.a) after the cell went through a designed cycling sequence that involves more than 20 electrochemical cycles at different rates and at a final discharged state of $3 \mathrm{~V}^{14}$. The schematics in Figure 1 illustrate our experimental approach. The cell was raster scanned in both $\mathrm{x}$ and $\mathrm{y}$ directions to collect multiple X-ray near edge structure (XANES) imaging datasets (Figure 1.b). We arbitrarily chose to scan 13 field-of-views that cover more than 100 particles (see labeled particles in supplementary Figure S1). After initial data reduction, we recovered over 10 million x-ray absorption spectra over the Co K-edge with each one of these spectra associated with a unique $\sim 30 \times 30 \mathrm{~nm}^{2}$ pixel. Due to the large amount of data acquired in our experiment, it is not possible to present all the spectra in a figure. We show in Figure 1.c a randomly selected subset (500) of the spectra to highlight the large quantity and complexity. We would point out that the counting statistics in these spectra (Figure 1.c) is not optimal partly because the measurements were done with time constraints as they were performed under operando conditions. The glassy carbon windows on the cell holder, the aluminum pouch, the Li metal anode, the separator and the liquid electrolyte all contribute to the background of the data, reducing the $\mathrm{x}$-ray transmission and further degrading the signal to noise ratio in the XANES spectra. As a result, direct applications of existing clustering algorithms such as $\mathrm{PCA}^{18}$ and DBSCAN $^{6}$ have very limited success in this case.

In order to enable the clustering calculation and to improve the accuracy and the robustness of our unsupervised search, we normalized the spectra (see supplementary Information) and extracted six key spectroscopic data attributes that denote important characteristics of the spectra. The six attributes are: (1) $\sigma$ : the standard deviation of the pre-edge data with respect to the linear regression line (supplementary Figure S2.c); (2) $\mathrm{H}_{\text {peak }}$ : the height of the white-line peak (supplementary Figure S2.d); (3) $\mathrm{E}_{\text {peak }}$ : the white-line peak energy (supplementary Figure S2.e);

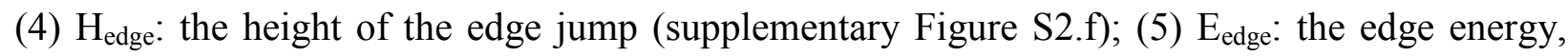


which is defined as the energy associated with the normalized intensity at 0.5 (supplementary Figure S2.g); and (6) $\chi^{2}$ : the goodness of the linear combination fit using the two known Co spectra expected in the cell (that of the $\mathrm{LiCoO}_{2}$ at charged (4.6V) and discharged (3V) states) (supplementary Figures S2.h and S4). These attributes are chosen because they have physical meanings relevant to the local chemistry, the quality of the spectrum or the degree to which that spectrum contains unanticipated information. For example, $\sigma$ is effectively a measurement of the noise level. This is because the spectrum's pre-edge region is known to be a smooth curve and any high frequency variation of the data in the pre-edge region is caused by noise associated with the measurement. $E_{\text {peak }}$ and $E_{\text {edge }}$ are associated with the valence state in many systems and have been used as the indicators of the relative state of charge in several studies ${ }^{19,20} \cdot \chi^{2}$ indicates the degree to which a conventional supervised approach ${ }^{16,21}$ would represent the spectrum, and is dependent on new chemical information contained in it, but also on the quality of the spectrum (dominantly the signal to noise ratio). The spatial distributions of these key data attributes over three selected regions of interest are shown in Figure 2. The top and the middle rows in Figure 2 contain the two particles (P37 and P46) with unanticipated chemical fingerprints; the bottom row in Figure 2 is a typical field view that contains normal $\mathrm{LiCoO}_{2}$ particles. The identification of these regions of interest is based on our data-mining approach, which will be discussed in details below.

These descriptors can be weighted differently before they are fed into the clustering calculation based on how much one would emphasize certain aspects of the data. For example, $\mathrm{H}_{\text {edge }}$ (supplementary Figure S2.f) is closely related to the density and thickness of the material that contains Co. It does not necessarily reflect the difference in chemical composition and can be, therefore, weighted less than the other descriptors. For more systematic evaluation of the contribution from different data attributes, we divided the six data attributes into two groups: the signal attributes $\left(\mathrm{H}_{\text {edge }}\right.$ and $\left.\sigma\right)$ and the chemical attributes $\left(\mathrm{H}_{\text {peak }}, \mathrm{E}_{\text {peak }}, \mathrm{E}_{\text {edge, }}\right.$, and $\left.\chi^{2}\right)$. On one hand, we weighted the chemical attributes more than the signal attributes because the chemical heterogeneity in the battery cell is our focus. On the other hand, the signal attributes are more useful when we screen the data points and reject pixels with insufficient signal to noise ratio.

It is also important to evaluate the degree of correlation among these data descriptors in order to ensure that we do not overemphasize some feature more than we planned and introduce unwanted redundancy. We show in supplementary Figure S3 the pairwise correlations among all the six attributes and a quantification of the correlation measured as Pearson correlation coefficient. The correlation analysis suggests that the data attributes we extracted are in general rather independent (see Supporting Information for more details).

After confirming the independency of all the six extracted data attributes, we implemented in our program several clustering algorithms including K-Means, agglomerative clustering, the spectral clustering, and DBSCAN (density-based spatial clustering of applications with noise) ${ }^{22}$. For this study, the result of K-Means clustering method is selected and presented because of the 
advantages of this particular algorithm including its scalability to very large dataset and relatively straightforward parameter settings.

Among all the particles scanned in our experiment, the clustering algorithm identified four clusters of pixels (the cluster maps are shown in Figures 3.b, 3d and 3.f; the corresponding spectra are shown in Figures 3.g and 3.h). The first two clusters are anticipated and cover over 98\% of the data, but cluster analysis identified two spectrally distinct particles (clusters $3 \#$ and $4 \#$ in P37 and P46, respectively). We would emphasize here that it is not possible to identify P37 and P46 as distinct from the rest of the particles by just analysis of each of the data attributes individually (see Figure 2), or by examination of the transmission images (see Figures 3.a, 3.c and 3.e for transmission images collected at $7850 \mathrm{eV}$ ). In Figures 3.a, 3.c and 3.e, we notice the highest contrast in P37, P46 and P14, which actually contain all the four different clusters. It again echoes our discussion regarding the importance of data attribute weighting for emphasizing the chemical heterogeneity rather than the density. As we show in the radar chart in Figure 3.i, the differences in the spectroscopic signatures of the unanticipated species are fully captured only by combining several of the extracted data attributes, which also highlights the effectiveness of our approach.

Co in both of the unanticipated new clusters is more reduced than designed for a normal operation of $\mathrm{LiCoO}_{2}$ cathode. But they point to two different pathways by which capacity degraded in a battery cell upon operation. Let us first focus on P46 (cluster \#4). The average Co edge XANES spectrum for cluster \#4 matches well the spectrum for Co metal. The direct observation of metallic Co in particle $\mathrm{P} 46$ confirms the common belief ${ }^{23,24}$ that at high charging voltage the transition metal dissolves out as $\mathrm{Co}$ oxide from the layered $\mathrm{LiCoO}_{2}$ cathode and is subsequently reduced at anode due to side reaction with electrolyte. Existence of the metallic Co particles in working cell can accelerate the growth of SEI on anode surface, consuming usable Li ions and aggravating the capacity loss during cycling.

Discovery of cluster \#3 in the center of P37, with more reduced Co at the core of the particles, indicates an alternate degradation mechanism. Existence of Co at lower oxidation state than $\mathrm{Co}^{3+}$ suggests that breakdown occurred due to local over-lithiation during discharge. However, considering that the cell was discharged (lithiated) to $3 \mathrm{~V}$ before the X-ray imaging measurement, the conventional wisdom suggests surface region of the particle should be more reduced than the core region, if the reaction inhomogeneity is caused by the $\mathrm{Li}^{+}$diffusion kinetics. The fact that the core region of the particle is more reduced indicates that the over-lithiation must have occurred before the final discharge, and the core has become electrochemically inactive. Overlithiation is known to transform layered $\mathrm{LiCoO}_{2}$ to $\mathrm{Li}_{1+\mathrm{x}} \mathrm{CoO}_{2-\mathrm{y}} / \mathrm{Li}_{2} \mathrm{O}$ micro composite ${ }^{25,26}$. This phase transition is associated with a large volumetric change, which is liable to form mechanical cracks to relieve stress. The formation of nano-cracks, not directly visible due to limited spatial resolution of the instrument, isolates the over-lithiated core from the rest of the particle, making it electrochemically inactive. On further cycling more and more over-lithiated micro domains went "dead" in P37 and eventually formed a large, electrochemically dead core at reduced Co 
valence state. The detailed illustration of the formation mechanism of P37 is schematically shown in Figure 4. We also summarize the possible pathways of the anticipated and unanticipated reactions in the supplementary Figure S5.

In conclusion, we have demonstrated the capability to collect and analyze over 10 million x-ray absorption spectra covering over 100 active particles in a $\mathrm{LiCoO}_{2}$ battery cell, as it undergoes fast charge-discharge cycles. The advanced data-mining approach was applied to these spectra and resulted in the discovery of two unanticipated minority phases. The discovery of these two minority phases and the determination of their locations provide direct evidence and insights into how battery cells lose capacity upon fast charge-discharge cycle. Although Co in both of the specified clusters are in more reduced state than expected, they reveal two different capacity fading mechanisms: The first particle (P46) provides evidence for a proposed degradation mechanism that dissolves Co cation and precipitates metallic Co as the local voltage exceeds the upper potential limit. The other particle (P37) suggests a more complicated degradation mechanism that involves the over-lithiation and deactivation of the local micro domains inside the $\mathrm{LiCoO}_{2}$ particle. These discoveries were made through analyzing huge amount of spectra, which would not be possible at all if the advanced data-mining techniques were not well developed. It is clear that the data-mining approach presented in this work will find great opportunities in a large number of research fields, well beyond the battery research field.

\section{Associated Content}

\section{Supporting Information}

Further details on Materials and Methods and supplementary figures S1-S5.

This material is available free of charge via the Internet at http://pubs.acs.org.

\section{Author Information}

\section{Corresponding Authors}

*Email: 1iuyijin@slac.stanford.edu (Y.L.)

*Email: xyu@iphy.ac.cn (X.Y.)

*Email: mehta@slac.stanford.edu (A.M.)

\section{Author Contributions}

${ }^{\perp}$ K.Z., F.R. and X.W. contributed equally to this work.

Y.L. and X.Y. conceived this work. E.H., Y.X, X.Y., and Y.L. performed the TXM measurements. X.W. and X.Y. did the characterization of the pristine material and prepared the battery pouch cell. K.Z., F.R., X.W., Y.L. analyzed the data and developed the data-mining approach. X.-Q.Y., H.L., A.M. and P.P. contributed to the interpretation of the spectromicroscopy data. K.Z., F.R., X.W., A.M., X.Y. and Y.L. prepared the manuscript with critical inputs from all the authors.

\section{Note}

The authors declare no competing financial interests. 


\section{Acknowledgement}

The support from D. Van Campen, V. Borzenets, D. Day and R. Davis for the experiments at beamlines 6-2C and 4-1 of SSRL is gratefully acknowledged. The authors gratefully thank Dr. J. Corbett of SLAC for valuable discussions. The work at IOP was supported by funding from Ministry of Science and Technology of China (Grants 2016YFB0100300) and Beijing Municipal Science \& Technology Commission (Grants D151100003115003). The work at Brookhaven National Laboratory was supported by the U.S. Department of Energy, the Assistant Secretary for Energy Efficiency and Renewable Energy, Office of Vehicle Technologies through Advanced Battery Material Research (BMR) program (Battery500 consortium) under Contract No. DE-SC0012704. This work was partly supported by the National Key Research and Development Program of China (Grant No. 2016YFA0400900), the National Natural Science Foundation of China (Grant No. 11535015, U1632110). Portion of this work is supported by Open Research Foundation of State Key Lab. of Digital Manufacturing Equipment \& Technology in Huazhong University of Science \& Technology. Use of the Stanford Synchrotron Radiation Lightsource, SLAC National Accelerator Laboratory, is supported by the U.S. Department of Energy, Office of Science, Office of Basic Energy Sciences under Contract No. DE-AC02-76SF00515.

\section{Reference}

(1) Lin, F.; Markus, I. M.; Nordlund, D.; Weng, T.-C.; Asta, M. D.; Xin, H. L.; Doeff, M. M. Nat. Commun. 2014, 5, 3529-3527.

(2) Yu, Y. S.; Kim, C.; Liu, Y.; Van Der Ven, A.; Meng, Y. S.; Kostecki, R.; Cabana, J. Adv. Energy Mater. 2015, 5, 14020401-14020408.

(3) Liu, Y.; Meirer, F.; Krest, C. M.; Webb, S.; Weckhuysen, B. M. Nat. Commun. 2016, 7, 1263412642.

(4) Zhang, Z.; Zhang, Q.; Shi, J.; Chu, Y. S.; Yu, X.; Xu, K.; Ge, M.; Yan, H.; Li, W.; Gu, L.; Hu, Y.-S.; Li, H.; Yang, X.-Q.; Chen, L.; Huang, X. Adv. Energy Mater. 2017, 7, 16011961-160119611.

(5) Zhou, Y. N.; Yue, J. L.; Hu, E.; Li, H.; Gu, L.; Nam, K. W.; Bak, S. M.; Yu, X.; Liu, J.; Bai, J. Adv. Energy Mater. 2016, 6, 16005971-16005978.

(6) Duan, X.; Yang, F.; Antono, E.; Yang, W.; Pianetta, P.; Ermon, S.; Mehta, A.; Liu, Y. Sci Rep. 2016, 6, 34406-34414.

(7) Lim, J.; Li, Y.; Alsem, D. H.; So, H.; Lee, S. C.; Bai, P.; Cogswell, D. A.; Liu, X.; Jin, N.; Yu, Y.-s. Science 2016, 353, 566-571.

(8) Wang, J.; Chen-Wiegart, Y.-c. K.; Eng, C.; Shen, Q.; Wang, J. Nat. Commun. 2016, 7, 12372-12379.

(9) Kuppan, S.; Xu, Y.; Liu, Y.; Chen, G. Nat. Commun. 2017, 8, 14309-14319.

(10) Bauer, S.; de Biasi, L.; Glatthaar, S.; Toukam, L.; Geßwein, H.; Baumbach, T. Phys. Chem. Chem. Phys. 2015, 17, 16388-16397.

(11) Farmand, M.; Celestre, R.; Denes, P.; Kilcoyne, A. D.; Marchesini, S.; Padmore, H.; Tyliszczak, T.; Warwick, T.; Shi, X.; Lee, J. Appl. Phys. Lett. 2017, 110, 063101-1-063101-10.

(12) Wolf, M.; May, B. M.; Cabana, J. Chem. Mater 2017, 29, 3347-3362.

(13) Nelson Weker, J.; Toney, M. F. Adv. Funct. Mater. 2015, 25, 1622-1637.

(14) Xu, Y.; Hu, E.; Zhang, K.; Wang, X.; Borzenets, V.; Sun, Z.; Pianetta, P.; Yu, X.; Liu, Y.; Yang, X.-Q. ACS Energy Letter 2017, 2, 1240-1245. 
(15) Liu, Y.; Andrews, J.; Meirer, F.; Mehta, A.; Gil, S. C.; Sciau, P.; Mester, Z.; Pianetta, P. In Applications of Hard X - ray Full - Field Transmission X - ray Microscopy at SSRL, The 10th International Conference on X-ray Microscopy, AIP Conference Proceedings, Chicago, Illinois, (USA), 2011; McNulty, I., Ed. AIP: Chicago, Illinois, (USA), 2011; pp 357-360.

(16) Meirer, F.; Cabana, J.; Liu, Y.; Mehta, A.; Andrews, J. C.; Pianetta, P. J.Synchrotron.Radiat. 2011, 18, 773-781.

(17) Meirer, F.; Liu, Y.; Pouyet, E.; Fayard, B.; Cotte, M.; Sanchez, C.; Andrews, J. C.; Mehta, A.; Sciau, P. J. Anal. At. Spectrom. 2013, 28, 1870-1883.

(18) Boesenberg, U.; Meirer, F.; Liu, Y.; Shukla, A. K.; Dell'Anna, R.; Tyliszczak, T.; Chen, G.; Andrews, J. C.; Richardson, T. J.; Kostecki, R. Chem. Mater. 2013, 25, 1664-1672.

(19) Xu, Y.; Hu, E.; Yang, F.; Corbett, J.; Sun, Z.; Lyu, Y.; Yu, X.; Liu, Y.; Yang, X.-Q.; Li, H. Nano Ener. 2016, 28, 164-171.

(20) Gent, W. E.; Li, Y.; Ahn, S.; Lim, J.; Liu, Y.; Wise, A. M.; Gopal, C. B.; Mueller, D. N.; Davis, R.; Weker, J. N. Adv. Mater. 2016, 28, 6631-6638.

(21) Nelson, G. J.; Harris, W. M.; Izzo Jr, J. R.; Grew, K. N.; Chiu, W. K.; Chu, Y. S.; Yi, J.; Andrews, J. C.; Liu, Y.; Pianetta, P. Appl. Phys. Lett. 2011, 98, 173109-1-173109-3.

(22) Pedregosa, F.; Varoquaux, G.; Gramfort, A.; Michel, V.; Thirion, B.; Grisel, O.; Blondel, M.; Prettenhofer, P.; Weiss, R.; Dubourg, V. J. Mach. Learn. Res. 2011, 12, 2825-2830.

(23) Zheng, H.; Sun, Q.; Liu, G.; Song, X.; Battaglia, V. S. J. Power Sources 2012, 207, 134-140.

(24) Komaba, S.; Kumagai, N.; Kataoka, Y. Electrochim. Acta 2002, 47, 1229-1239.

(25) Shu, J.; Shui, M.; Huang, F.; Ren, Y.; Wang, Q.; Xu, D.; Hou, L. J. Phys. Chem. C. 2010, 114, 3323-3328.

(26) Chadwick, A. V.; Savin, S. L.; Alcántara, R.; Fernández Lisbona, D.; Lavela, P.; Ortiz, G. F.; Tirado, J. L. ChemPhysChem 2006, 7, 1086-1091.

(27) Liu, Y.; Meirer, F.; Wang, J.; Requena, G.; Williams, P.; Nelson, J.; Mehta, A.; Andrews, J. C.; Pianetta, P. Anal. Bioanal. Chem. 2012, 404, 1297-1301.

(28) Weng, T.-C.; Waldo, G. S.; Penner-Hahn, J. E. J.Synchrotron.Radiat. 2005, 12, 506-510.

(29) Liu, Y.; Meirer, F.; Williams, P. A.; Wang, J.; Andrews, J. C.; Pianetta, P. J.Synchrotron.Radiat. 2012, 19, 281-287. 


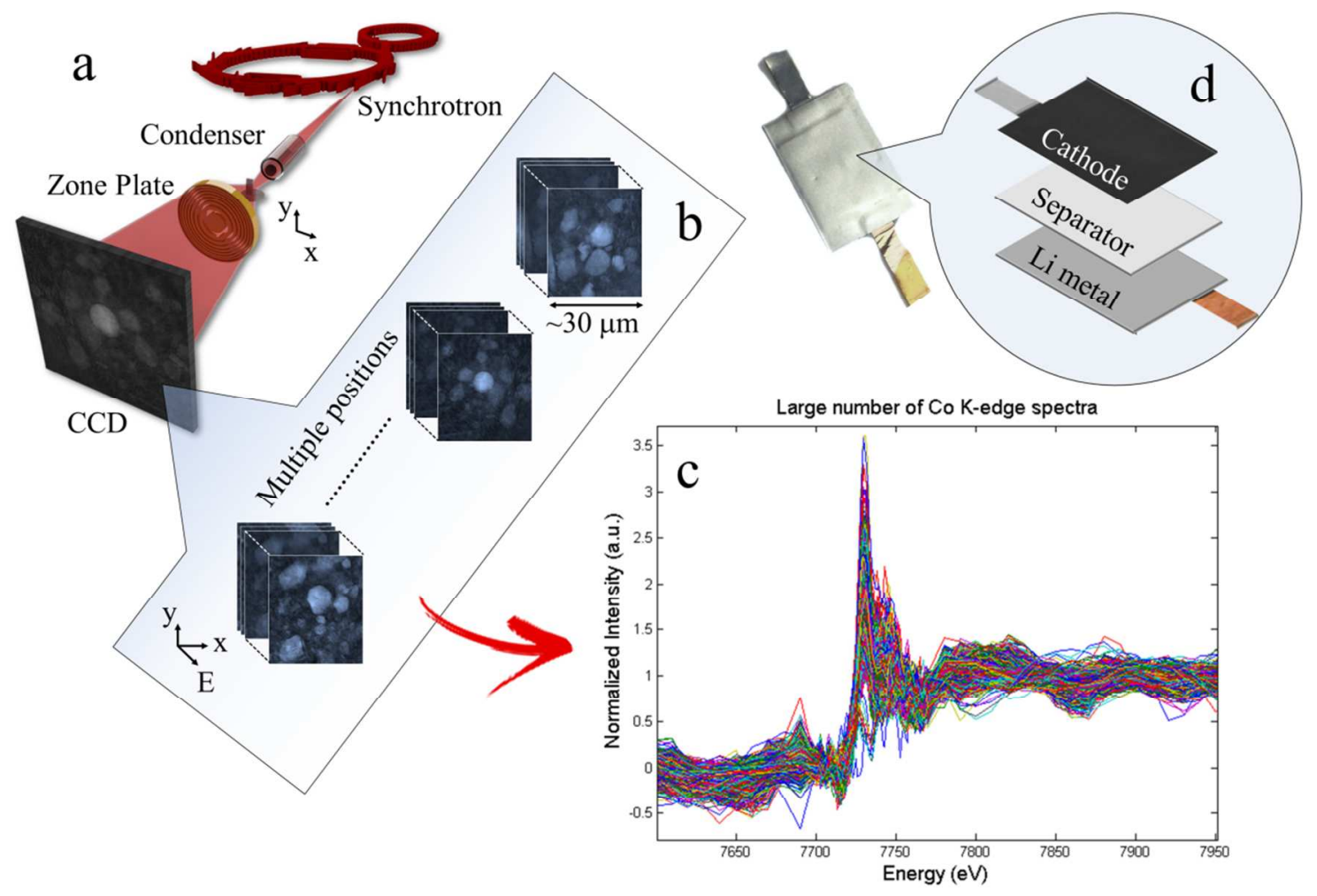

Figure 1. Schematics of the experimental approach. Panel (a) is the experimental setup. The sample was raster scanned in both $\mathrm{x}$ and $\mathrm{y}$ directions. (b) Spectro-microscopic datasets are acquired at 13 different positions on cell covering more than 100 particles with over 10 million $\mathrm{x}$-ray absorption spectra over the Co K-edge. A randomly chosen subset (500) of the spectra is shown in panel (c) to highlight the large data quantity and complexity. Panel (d) shows the structure of the half-cell used in this study. 

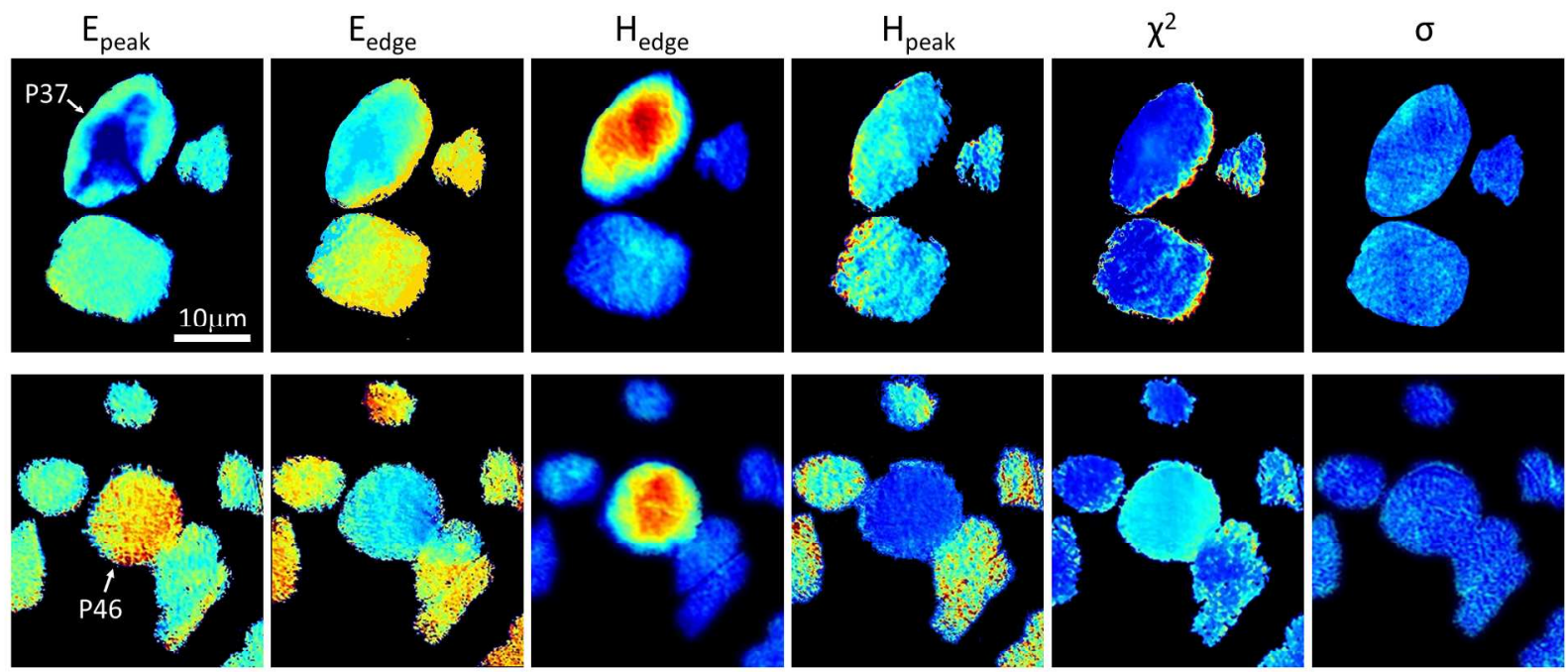
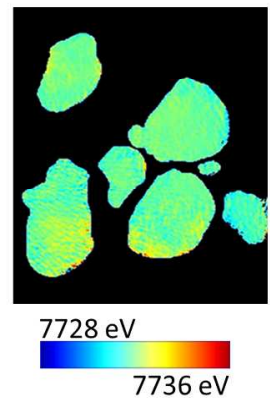

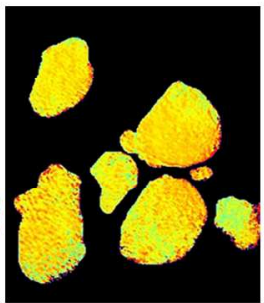

$7711 \mathrm{eV}$ $7724 \mathrm{eV}$

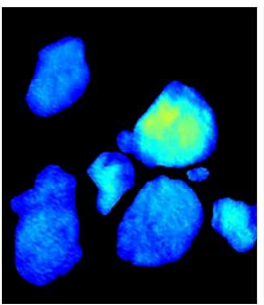

0

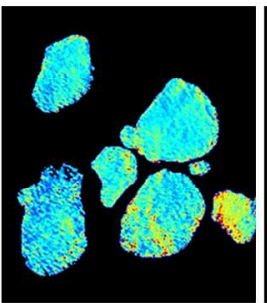

$-0.3$

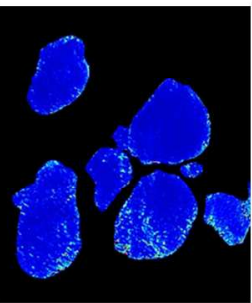

$-0.01$

0.7
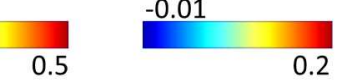

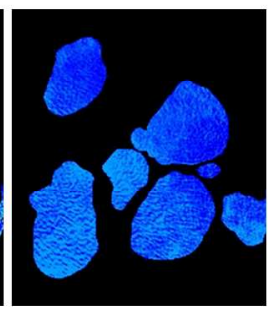

0.006

Figure 2. The spatial distribution of the extracted data attributes (supplementary Figure S2) over selected regions of interest. The top and the middle rows contain the unanticipated minority phases (P37 and P46 as labeled in the first column and in the supplementary Figure S1) identified by our method. The bottom row is a typical field of view that contains normal $\mathrm{LiCoO}_{2}$ particles. 

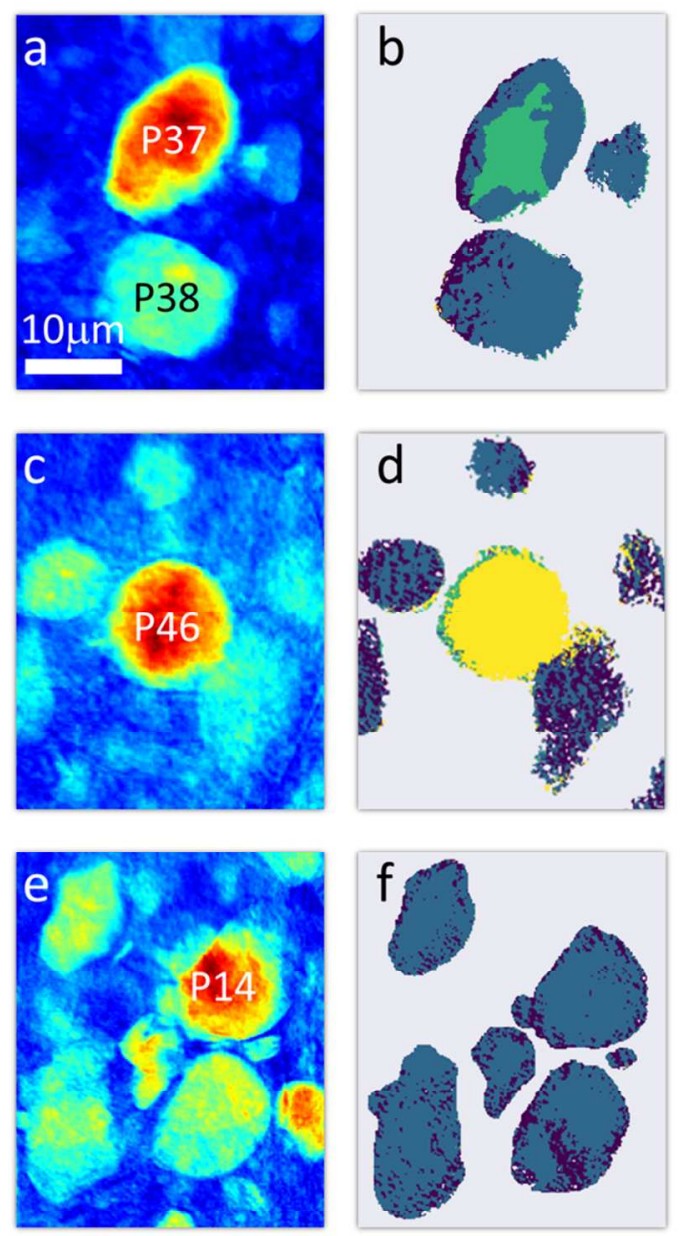

absorbance

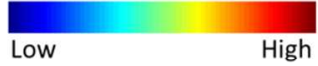

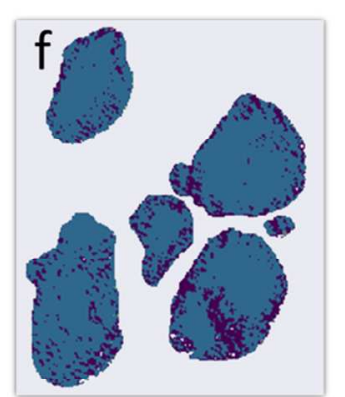

Cluster labels

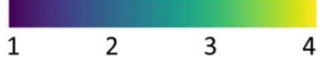

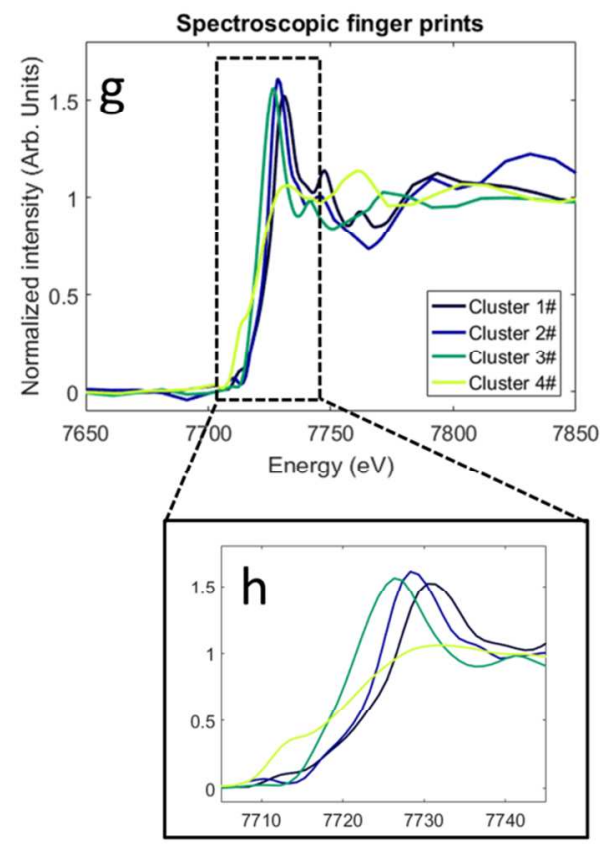

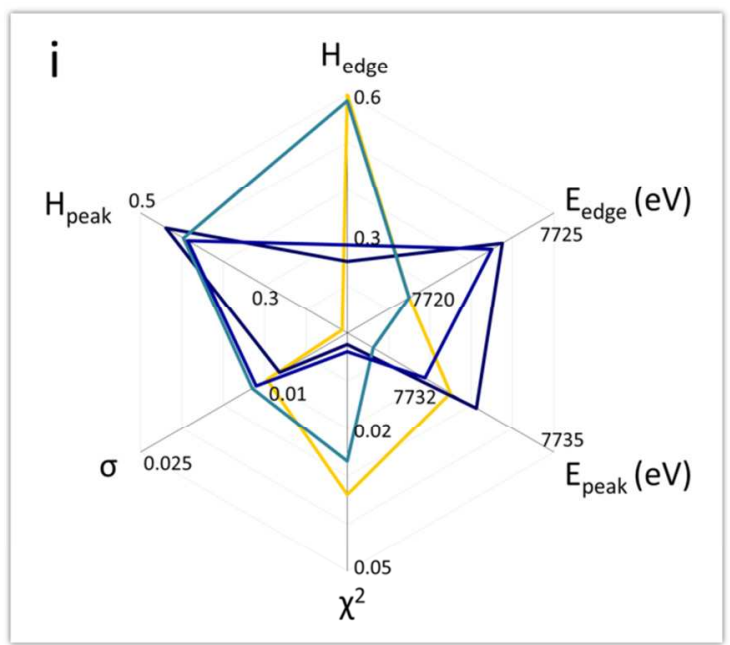

Figure 3. The minority phases identified by our data-mining approach. Panels (a) and (c) show the transmission images of the field-of-views covering particles (P37 and P46), which are identified by our method as minority phases. Panel (e) is the transmission image of another typical field-of-view that contains several normal $\mathrm{LiCoO}_{2}$ particles. Panels (b), (d), and (f) show the clustering results, with the four different clusters color coded to the corresponding inset. We compare the spectroscopic fingerprints of all the four clusters in panels (g) and (h). It is interesting to note that clusters $1 \#$ and $2 \#$ are both similar to $\mathrm{LiCoO}_{2}$ (see supplementary Figure S4). The radar chart in panel (i) shows the differences in the spectroscopic fingerprints of all the 4 clusters are systematically captured by the data attributes extracted in our approach. The scale bar in panel (a) is 10 microns. 


\section{Cycling Time}

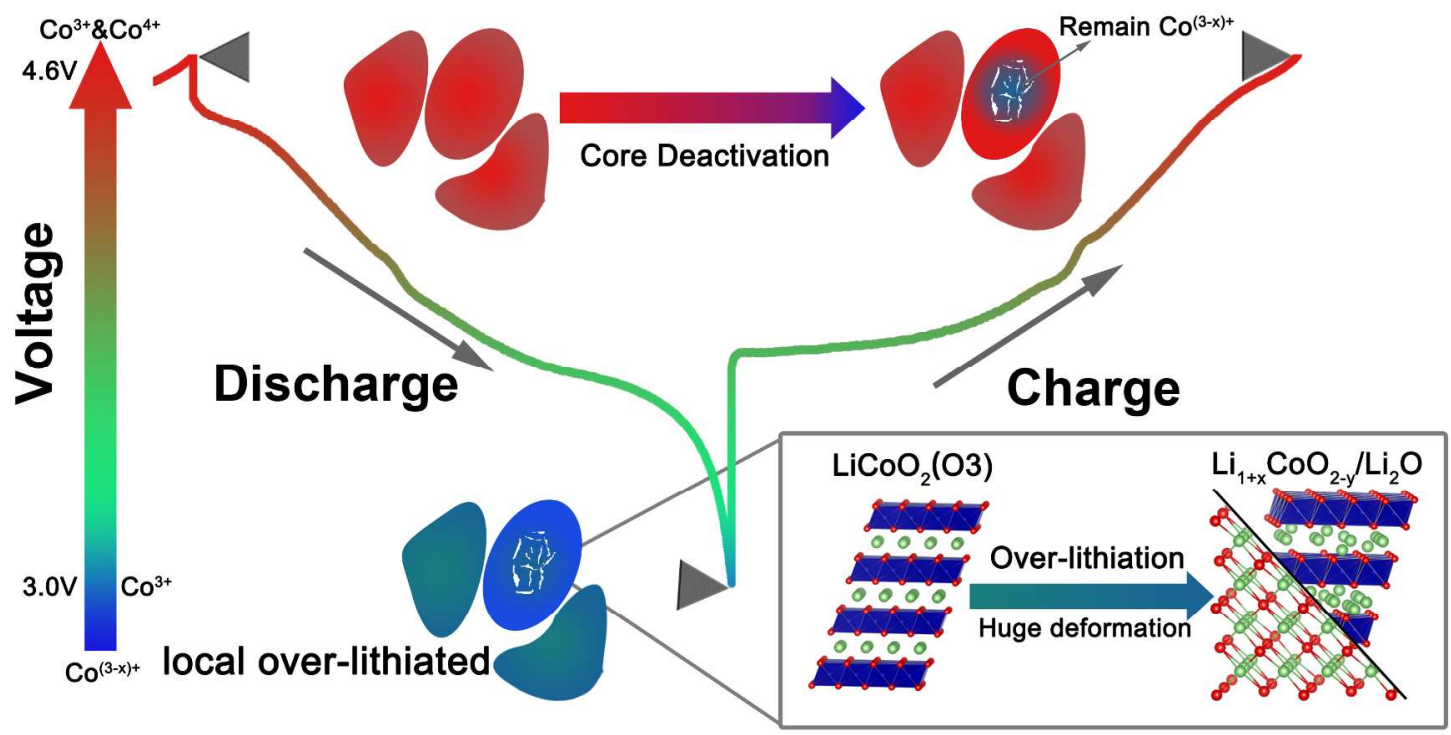

Figure 4. The schematic illustration of particle P37 degradation mechanism. The red and blue figures refer to the $\mathrm{LiCoO}_{2}$ particle at charged and discharged state. The electrochemical curve is posed to show the certain set where critical deformation occurred. The inset picture shows the comparison of standard and over-lithiated $\mathrm{LiCoO}_{2}$. The red and green spheres represent oxygen and lithium ions and the dark blue octahedron stands for $\mathrm{CoO}_{6}$ unit. 


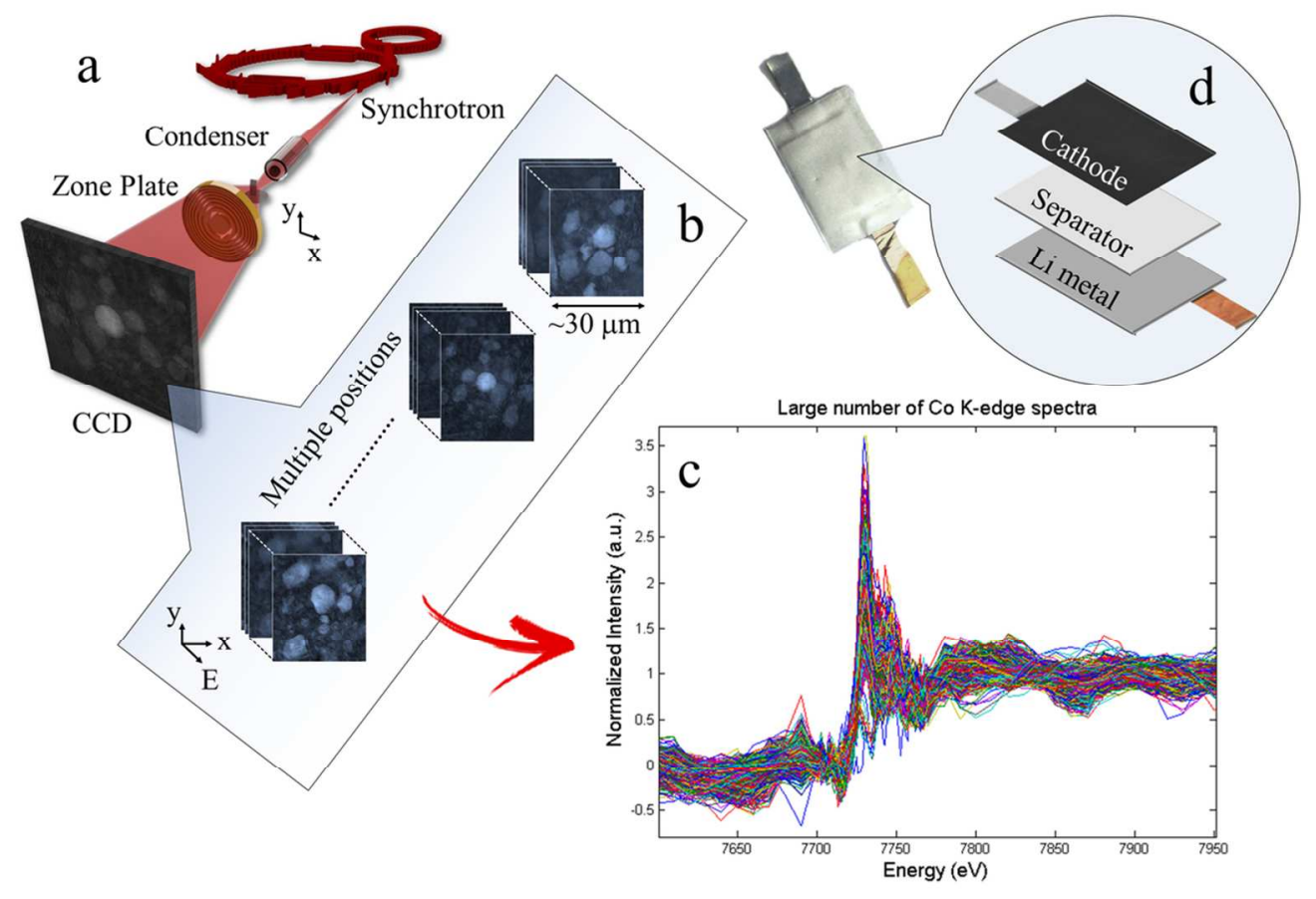

$103 \times 70 \mathrm{~mm}(300 \times 300 \mathrm{DPI})$ 

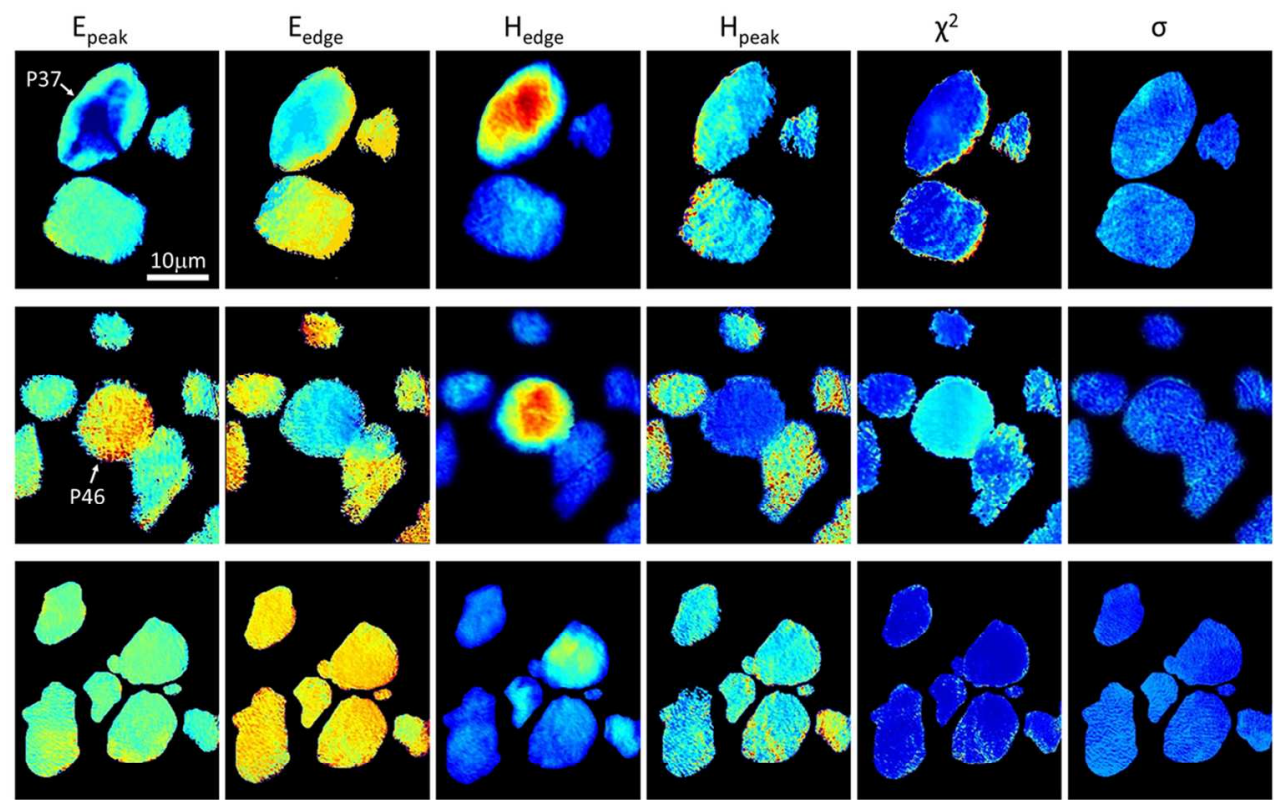

$7728 \mathrm{eV}$
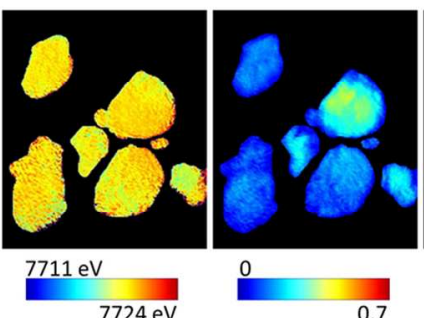

0

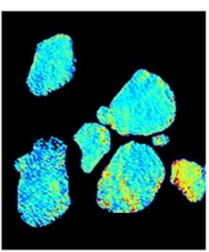

$-0.3$

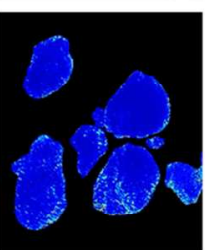

$-0.01$

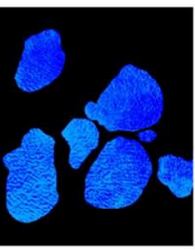

0.006

0.7
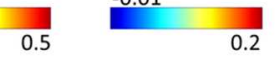

0.035

$104 \times 72 \mathrm{~mm}(300 \times 300 \mathrm{DPI})$ 

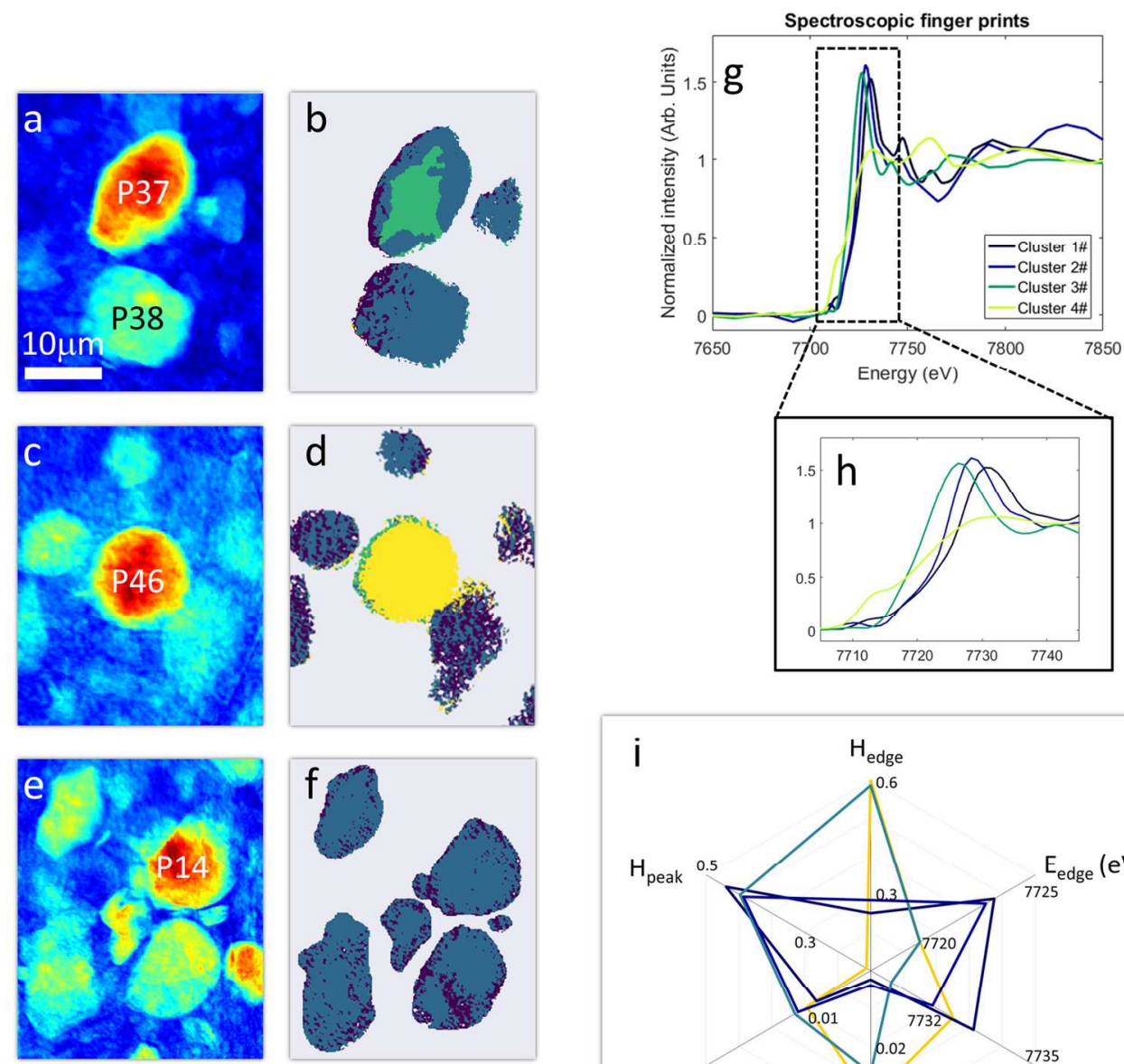

absorbance

Cluster labels
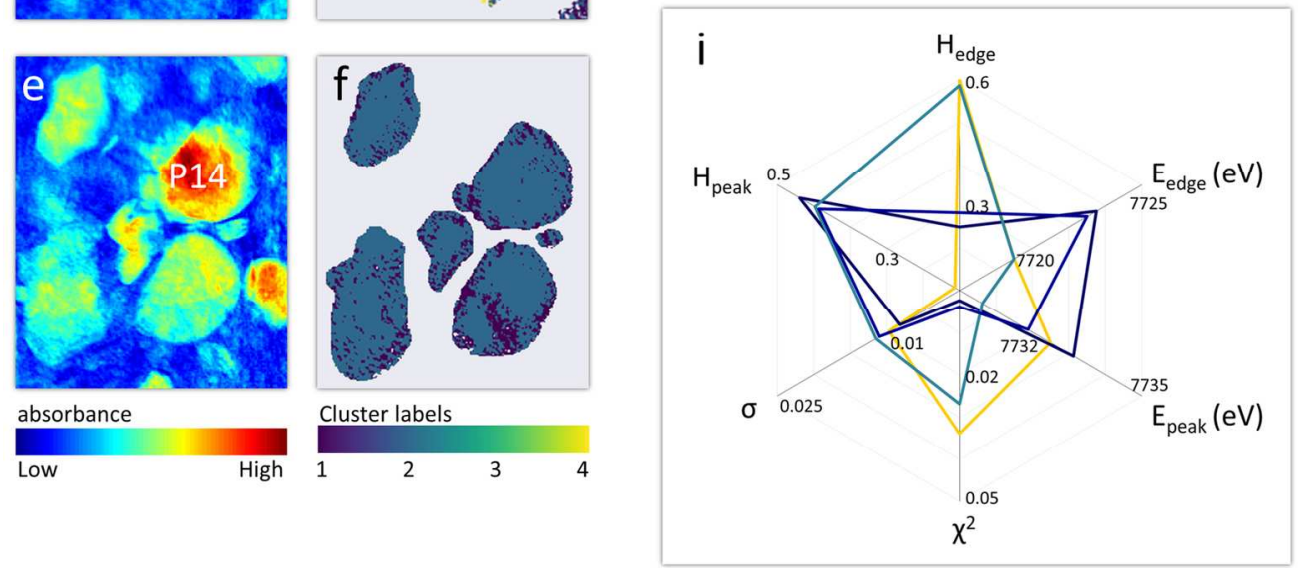

$157 \times 165 \mathrm{~mm}(300 \times 300$ DPI $)$ 


\section{Cycling Time}

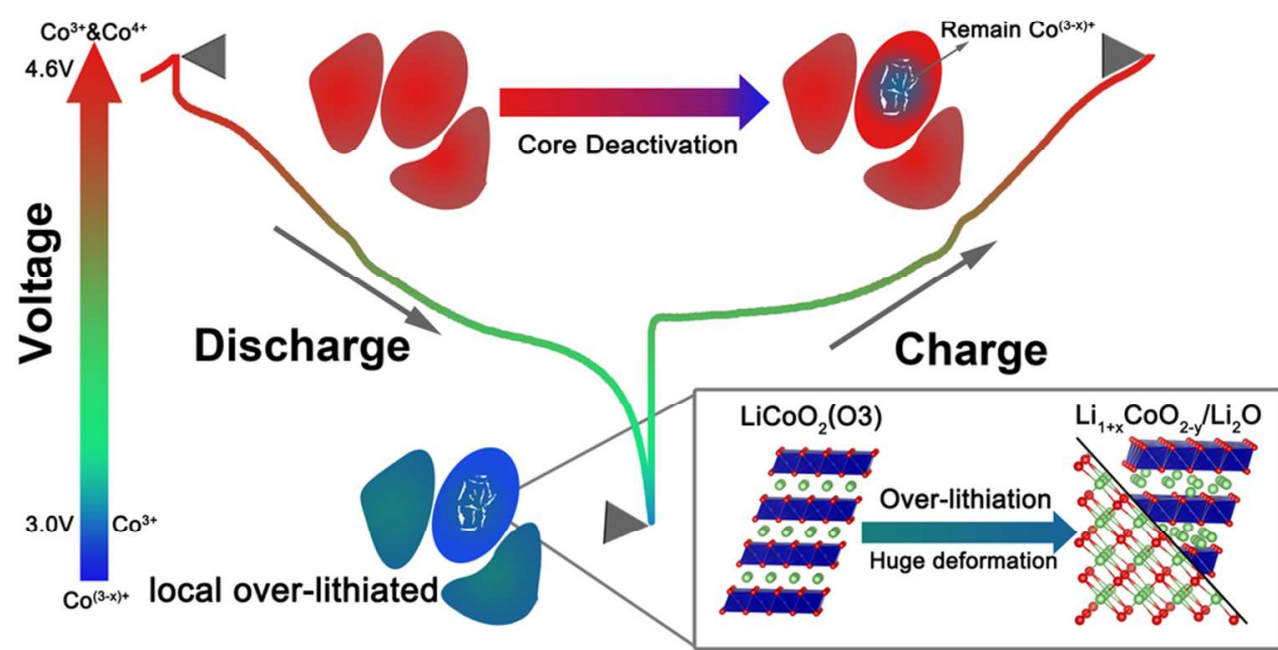

$87 \times 51 \mathrm{~mm}(300 \times 300$ DPI $)$ 
Big data collection

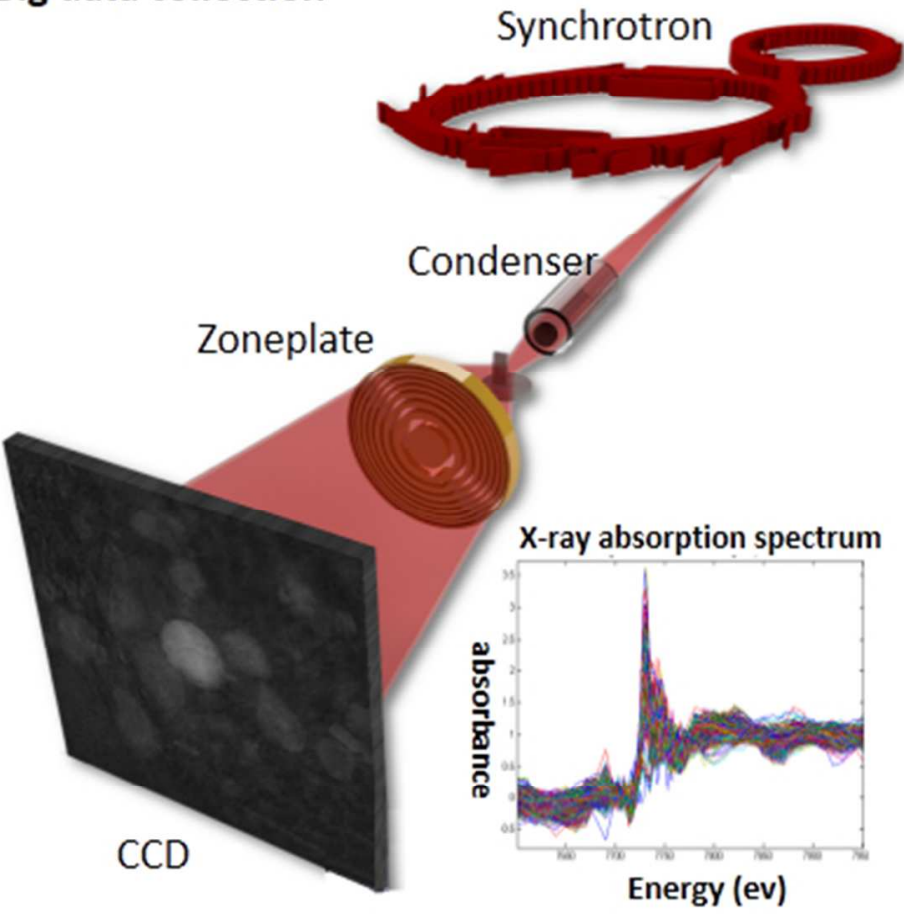

\section{Data Mining}

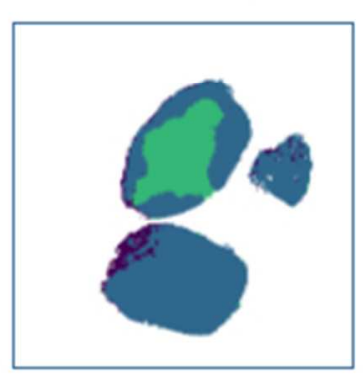

Scientific results

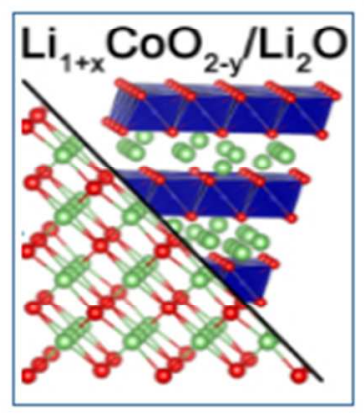

$47 \times 34 \mathrm{~mm}(300 \times 300 \mathrm{DPI})$ 


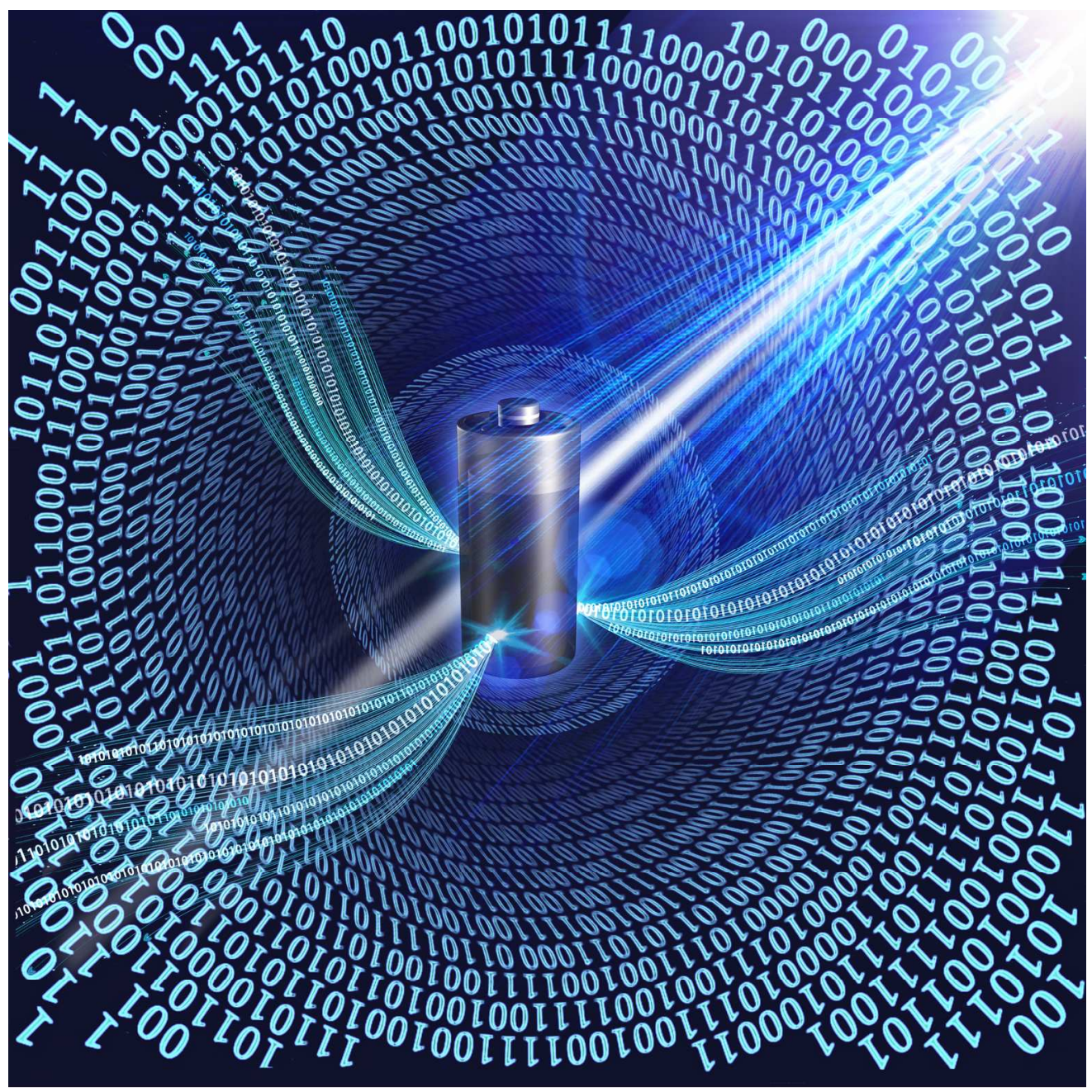

$199 \times 199 \mathrm{~mm}(300 \times 300$ DPI $)$ 